\title{
Wide field-of-view optical broadcasting for bi-directional indoor optical wireless communications employing PAM-4 modulation
}

\author{
Feng Feng, ${ }^{1, *}$ Paramin Sangwongngam, ${ }^{2}$ Grahame Faulkner, ${ }^{2}$ and Dominic O’Brien ${ }^{2}$ \\ ${ }^{1}$ Key Laboratory of Opto-electronics Information Technology of Ministry of Education and Key Laboratory of Integrated Opto-electronic \\ Technologies and Devices in Tianjin, School of Precision Instruments and Opto-electronics Engineering, Tianjin University, Tianjin 300072, China \\ ${ }^{2}$ Department of Engineering Science, University of Oxford, Parks Road, OX1 3PJ, Oxford, UK \\ ${ }^{*}$ Corresponding author: fengfeng.photon@yahoo.com
}

Received 5 August 2019; revised 16 October 2019; accepted 28 October 2019; posted 29 October 2019 (Doc. ID 374598); published 9 December 2019

\begin{abstract}
We present wide field-of-view (FOV) bi-directional pointto-multipoint indoor optical wireless communications operating over a range of $4 \mathrm{~m}$. The system is designed to integrate with fiber-to-the-home/building networks realized by a passive optical network. A phase-only spatial light modulator (SLM)-based beam steering base station with a $\pm 30^{\circ}$ FOV broadcasts downstream transmissions to two nomadic user terminals that use mirror-based beam steering to provide a $\pm 50^{\circ} \mathrm{FOV}$. At the base station, a composite phase mask is constructed on the SLM not only to perform optical broadcasting, but also to steer upstream optical transmissions from user terminals at a different wavelength. Successful upstream and downstream data transmission of 25 Gbit/s PAM4 is achieved.
\end{abstract}

Published by The Optical Society under the terms of the Creative Commons Attribution 4.0 License. Further distribution of this work must maintain attribution to the author(s) and the published article's title, journal citation, and DOI.

https://doi.org/10.1364/OL.44.006009

The demand for high-capacity wireless communications is growing very rapidly, in particular in indoor environments. This is stimulated by exponentially increasing wireless traffic generated indoors by an increasing number of broadband mobile devices, as well as the devices for the Internet of Things and emerging bandwidth-hungry multimedia applications, such as virtual reality and augmented reality. Traffic growth is congesting current wireless networks and exhausting the available radio spectrum. As an attractive solution, optical wireless communications (OWC) can use a vast unlicensed optical domain of wireless communication resources $[1,2]$. Meanwhile, fiber-to-the-home/ building (FTTH/B) networks enabled by passive optical network (PON) technologies can unlock terahertz scale bandwidths accessible to end users [3]. OWC using beam steered infrared narrow beams coupled directly from optical fiber networks can provide high-capacity wireless communications beyond
$10 \mathrm{Gbit} / \mathrm{s}$, and there is a wide range of choice of optical components available from the well-established fiber telecommunication industry. For OWC using infrared beams with wavelengths longer than $1400 \mathrm{~nm}$, eye safety standards are less stringent than at visible wavelengths $[4,5]$ making this wavelength region very attractive for communications. For $\lambda>1400 \mathrm{~nm}$, transmitting power up to $10 \mathrm{~mW}$ is allowed within the eye safety limit [1], compared with the allowed blue light power of $1.01 \mu \mathrm{W}$ in a white light communication system [5]. Nevertheless, there are substantial implementation challenges to be addressed. Owing to the very narrow field of view (FOV) of single-mode fiber-based components, optical beam steering is required at the fiber-based transmitter and receiver to provide a wide FOV, and it enables the implementation of tracking and localization functionalities. This has been demonstrated in a tracked point-to-point indoor OWC link [6]. To support a large number of user terminals in OWC systems effectively, wide FOV optical broadcasting (point-to-multipoint) is desired further from point-to-point links. We presented our initial results on wide FOV optical broadcasting in Ref. [7].

In this Letter, we describe many further details and demonstrate wide FOV optical broadcasting from a spatial light modulator (SLM)-based beam steering base station to two nomadic user terminals that use mirror-based beam steering in a bi-directional indoor OWC system over a range of $4 \mathrm{~m}$. The OWC system is designed to integrate with local optical fiber networks connected to PON enabled FTTH/B networks. The SLM-based base station has a $\pm 30^{\circ} \mathrm{FOV}$, and it broadcasts infrared light at a designated wavelength from an optical fiber to nomadic user terminals. The user terminals use a mirror-based beam steering with a $\pm 50^{\circ} \mathrm{FOV}$. In the upstream direction (from the user terminal to the base station), transmitted light from an optical fiber at a different wavelength travels along the same free space optical path with the downstream transmission. A composite phase mask is constructed at the SLM-based base station not only to broadcast the downstream optical transmissions from an optical fiber, but also to steer the upstream optical transmissions back to the fiber. This significantly improves the optical coupling loss of the 
upstream/downstream optical transmissions using a different wavelength, compared with a composite phase mask that only performs downstream/upstream optical broadcasting. This is because a liquid-crystal SLM gives different beam steering angles at different wavelengths. In the data transmission tests on the OWC system, we employ spectrum-efficient pulse amplitude modulation with four levels (PAM-4) for its low complexity and power consumption, and have experimentally demonstrated $25 \mathrm{Gbit} / \mathrm{s}$ data transmissions in both the downstream and upstream optical transmissions.

The bi-directional point-to-multipoint indoor OWC system is designed to integrate with FTTH/B networks enabled by time-division multiplexed (TDM) PONs, which broadcasts all optically the data-carrying time frames to the desired user terminals. As summarized in Ref. [8], different PON systems are standardized to operate at their allocated wavelength bands. Here we target the OWC system to operate at the wavelength bands planned for the second next generation PON (NGPON2). That is, the upstream and downstream wavelength bands, respectively, are located in the C-band (1524-1544 nm) and L-band (1596-1603 nm) [8]. Figure 1 shows the system design architecture. The SLM-based (15 $\mu \mathrm{m}$ pixel pitch, Meadowlark HSP512-1550) base station has a $\pm 30^{\circ}$ FOV at $1550 \mathrm{~nm}$, which is gained by $10 \times$ angle magnification of the SLM steering range $\left( \pm 3^{\circ}\right)$ using a telescope lens system. The steering range of an SLM increases with a smaller SLM pixel pitch and longer operating wavelength [9]. An increased FOV would be available with small pixel pitch SLM devices. Such multiple SLM-based base stations can be installed per room to provide the full coverage of a room, as well as backup links, while one beam from a base station is blocked. At user terminals, we use mirror-based beam steering. The dual axis steering mirrors (Optotune, MR-15-30-G) at user terminals are accurately controlled by electronics and have up to $\pm 50^{\circ}$ optical deflection from each axis and a steering resolution less than $5 \mu \mathrm{rad}$. Tracking and localization are important to make such OWC systems practical. We propose a tracking and localization scheme as illustrated in Fig. 1. A cost-effective near-infrared camera with a wide FOV at the base station tracks the locations of individually recognizable infrared "tags" placed on the user terminals simultaneously. At a user terminal, a position sensitive detector tracks the angle of arrival of the directed communication signal from the base station, using about a tenth of the receiver optical power. Circulators are employed

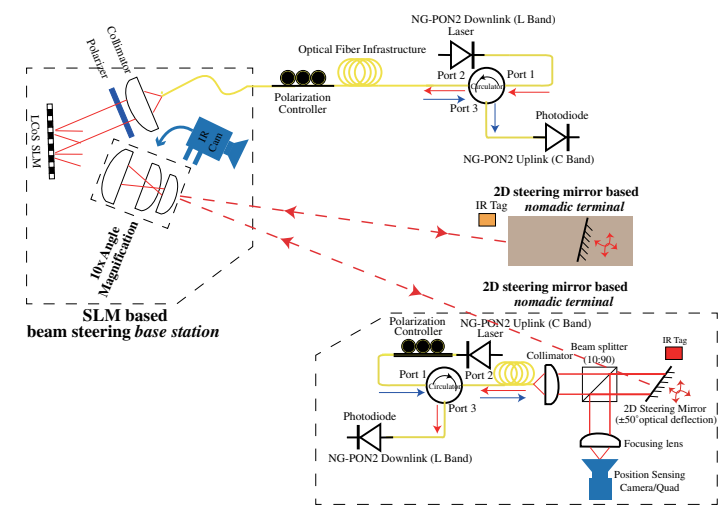

Fig. 1. System design of the wide FOV point-to-multipoint indoor bi-directional OWC system to integrate with FTTH/B networks enabled by NG-PON2. at the base station and nomadic user terminals for supporting bi-directional operation. The polarizations of the downstream and upstream optical transmissions are aligned by polarization controllers at the SLM-based base station and mirror-based user terminals to the polarization required by the SLM.

A liquid-crystal SLM is dispersive and gives different steering angles at different operating wavelengths. Therefore, as illustrated in Figs. 2(a) and 2(b), a single downstream/upstream beam steering phase mask on the SLM would cause misalignment (additional optical coupling loss) to the upstream/ downstream optical transmission, due to the different operating wavelengths of the downstream and upstream optical transmissions. In the rest of this Letter, we refer to this additional optical coupling loss as bi-directional operation coupling loss. We measured the bi-directional operation coupling loss to upstream/downstream transmission resulting from a single downstream/upstream phase mask on the SLM at different upstream and downstream operating wavelengths. The right parts of Figs. 2(a) and 2(b), respectively, show the measured bi-directional operation coupling loss to upstream and downstream transmissions versus different upstream and downstream wavelengths. We observe that for our optical system the bi-directional operation coupling loss resulting from a single upstream/downstream phase mask in the upstream and downstream operating wavelength bands is quite significant. As the separation of the downstream and upstream wavelength increases, the upstream/downstream steering angle deviation resulting from a single downstream/upstream phase mask becomes bigger, inducing more bi-directional operation coupling loss. As a result of a single downstream and upstream phase mask on the SLM, the bi-directional operation coupling loss to the upstream transmission ranges from 6.7 to $10.5 \mathrm{~dB}$, and the bi-directional operation coupling loss to the

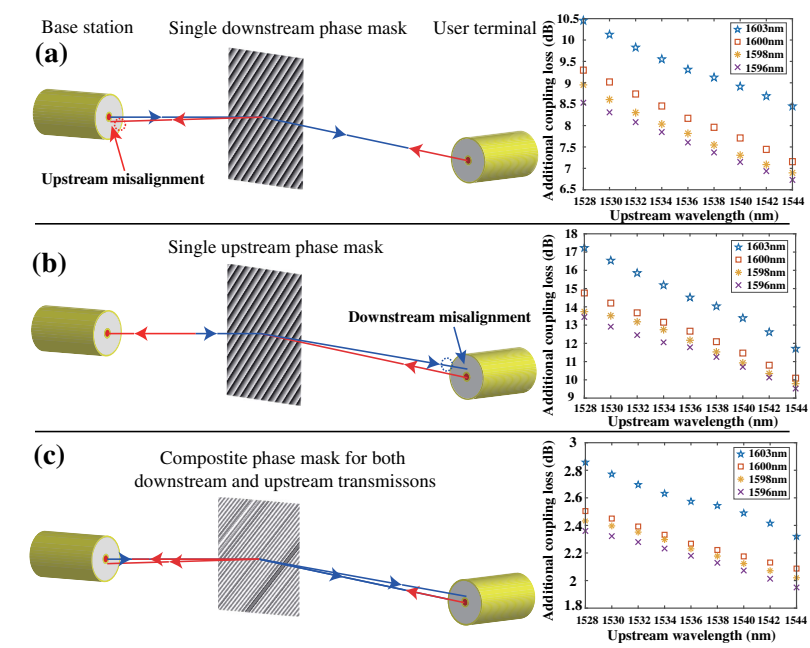

Fig. 2. Left, illustrations of bi-directional operations with different phase masks on the SLM. Right, the induced bi-directional operation coupling loss to corresponding upstream and/or downstream transmis$\operatorname{sion}(\mathrm{s})$ versus the upstream and downstream operating wavelengths. (a) With a single downstream phase mask, the upstream transmission experiences the additional loss. (b) With a single upstream phase mask, the downstream transmission experiences the additional loss. (c) With a composite phase mask for both upstream and downstream transmissions, both the upstream and downstream transmissions experience a relatively low additional coupling loss. 
downstream transmission ranges from 9.5 to $17.2 \mathrm{~dB}$. Our solution to this is to construct a composite phase mask for bi-directional operation between the base station and one user terminal by superposition of different downstream and upstream phase masks. As illustrated in Fig. 2(c), a composite phase mask for the bi-directional operation between the base station and a user terminal will split both of the downstream and upstream optical transmissions in two, one that is well aligned and the other that experiences a bi-directional operation coupling loss. This approach introduces up to $3 \mathrm{~dB}$ additional coupling loss to both the downstream and upstream transmissions of a bi-directional link between the base station and a user terminal, compared with a single upstream or downstream phase mask that causes a significant additional coupling loss to either the downstream or the upstream transmission. The right part of Fig. 2(c) shows the measured bi-directional operation coupling loss to both the downstream and the upstream transmissions using the bi-directional composite phase masks versus different operating wavelengths. We can see that, by using this approach, the bi-directional operation coupling loss to the downstream and upstream transmissions increases with the wavelength separation and ranges from 1.9 to $2.8 \mathrm{~dB}$. This marks a significant coupling loss improvement to the bi-directional transmissions. Considering tracking, the upstream steering angle deviations from the downstream link that is established by the tracking system can be calibrated using the downstream and upstream operating wavelengths.

To perform optical broadcasting, a composite phase mask for all user terminals that operate bi-directionally is needed. A composite phase mask performing optical broadcasting can be constructed by superposition of weighted beam steering phase masks for both downstream and upstream transmissions of each user terminals, as in Eq. (1). A similar methodology is used to construct composite phase masks to (de)multiplex multiple spatial modes in a multimode optical fiber [10]. This method allows accurate optical broadcasting to be performed without complex calculations with the terminal locations provided by the tracking system, compared with an iterative phase mask search approach [11]:

$$
h_{x, y}=\exp \left(i \cdot \arg \left[\sum_{m=0}^{M-1} w_{m} \cdot \theta_{m, x, y}\right]\right) \text {, }
$$

where $x, y$ are the pixel indices of a phase mask, $M$ is the total number of upstream and downstream transmission channels, $w_{m}$ is the weighting factor of channel $m$ to adjust relative power, and $\theta_{m}$ is the wavefront information of channel $m$. Here the wavefront information $\theta_{m}$ only refers to the beam steering angles. Aberation correction information to further improve optical coupling efficiency can also be included [12]. Due to the superposition nature of the broadcasting phase mask construction, updating the phase mask for location changes of a user terminal will not cause any link misalignment to other user terminals. The weighting factors can be re-optimized to adjust relative power after updating the phase mask when necessary. Figure 3(a) shows an example of constructed optical broadcasting composite phase masks for the case of two user terminals with an upstream wavelength of $1536 \mathrm{~nm}$ and a downstream wavelength of $1599 \mathrm{~nm}$. In the replay field (downstream), there are two pairs of closely overlapped Gaussian spots. As the separation of the downstream and upstream wavelengths increases, the overlapped Gaussian spots start separating from each other. Figure 3(b) shows the replay field for a downstream wavelength of $1603 \mathrm{~nm}$ and an upstream wavelength of $1524 \mathrm{~nm}$ which corresponds to the largest upstream and downstream wavelength separation specified in the NG-PON2 standard. Here the two overlapped spots are almost apart from each other, also indicated by the $2.8 \mathrm{~dB}$ bi-directional operation coupling loss shown in Fig. 2(c). Figure 3(c) shows the replay field for a downstream wavelength of $1577 \mathrm{~nm}$ and an upstream wavelength of $1270 \mathrm{~nm}$, with wavelengths specified in the XG-PON standard [8]. For this case, the two overlapped spots have been completely separated. This would induce a $3 \mathrm{~dB}$ additional coupling loss resulting from the bi-directional operation. In this Letter, we only demonstrate optical broadcasting to two user terminals. More user terminals can be supported by superposing more beam steering phase masks at the cost of extra splitting loss. The maximum number of user terminals that can be supported is mainly determined by the total loss of a link and power budget of transceiver systems used. We generalize the bi-directional optical link loss between the base station and one user terminal for the case of broadcasting to $N$ user terminals in Eq. (2):

$$
\begin{aligned}
\operatorname{Loss}_{\text {Total of one link }}(\mathrm{dB})= & \operatorname{Loss}_{\text {Avg_coupling }}-10 \log _{10}\left(\frac{1}{N}\right) \\
& +\operatorname{Loss}_{\text {Bi-directional. }}
\end{aligned}
$$

The $N$ broadcast bi-directional links have almost the same geometric loss as they use the same optical system, but experience varied diffraction efficiencies across the replay field [9] and different optical aberrations from the angle magnification lens system due to varying offsets from the optical axis of the lens system. The weighting factors of all the bi-directional transmissions in Eq. (1) can be adjusted through an iterative feedback loop to equalize the losses, resulting in Loss Avg_coupling. $_{\text {. }}$. Additionally, the optical broadcasting introduces splitting loss, which is reflected in the term $10 \log _{10}\left(\frac{1}{N}\right)$ in Eq. (2). The total optical loss of a bi-directional link between the base station and one of the $N$ user terminals also needs to take account of the bi-directional operation coupling loss, Loss $_{\mathrm{Bi} \text {-directional }}$.

An experimental demonstration of data transmission for a two user terminal-based system was undertaken, as shown in Fig. 4(a). The two mirror-based user terminals are angularly

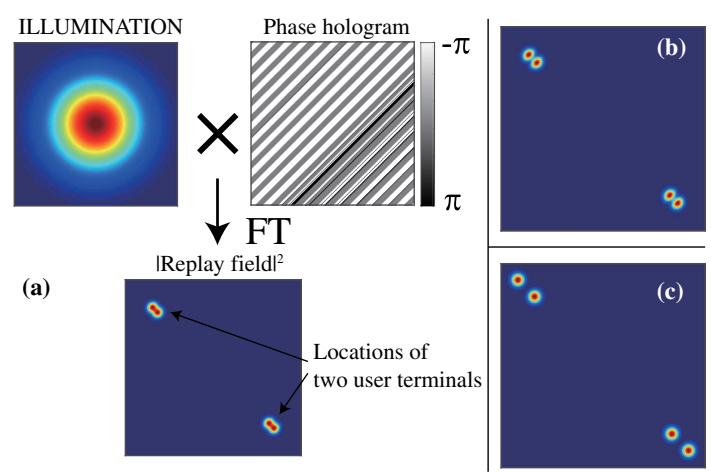

Fig. 3. (a) Illustrative example of the bi-directional optical broadcasting to two user terminals with a $1599 \mathrm{~nm}$ downstream wavelength and $1536 \mathrm{~nm}$ upstream wavelength. (b) Downstream replay field with $1603 \mathrm{~nm}$ downstream wavelength and $1524 \mathrm{~nm}$ upstream wavelength. (c) Downstream replay field with $1577 \mathrm{~nm}$ downstream wavelength and $1270 \mathrm{~nm}$ upstream wavelength. 

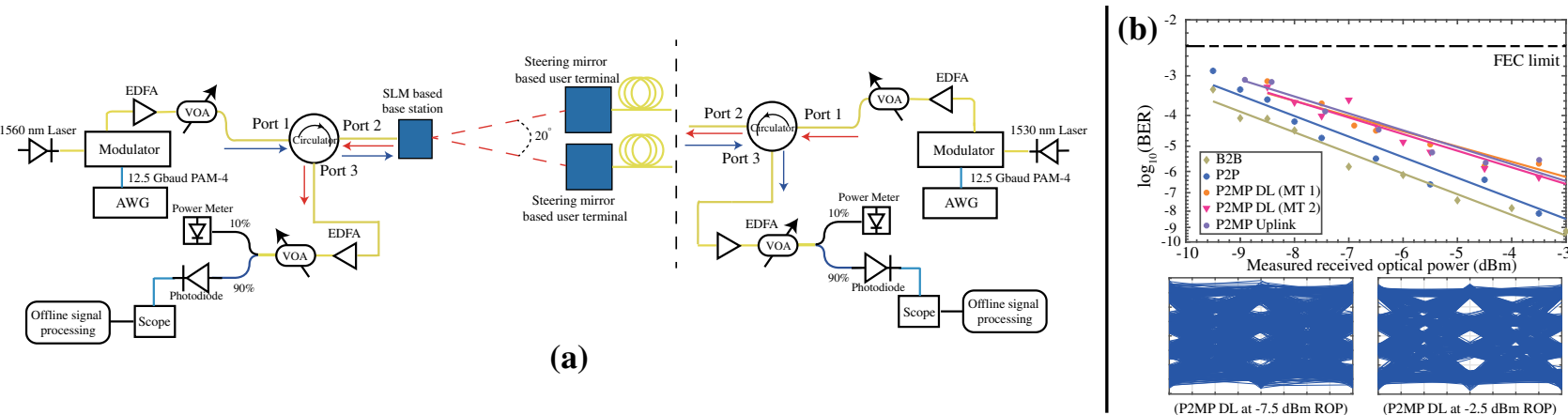

Fig. 4. (a) Experimental system for the wide FOV bi-directional point-to-multipoint data transmission tests using PAM-4. (b) BER curves for the broadcast bi-directional transmissions at 25 Gbit/s using PAM-4 and typical eye diagrams. ROP, received optical power.

separated by approximately $20^{\circ}$ at a distance of $4 \mathrm{~m}$ from the base station. For experiments, the lack of an L-band EDFA led to the selection of 1560 and $1530 \mathrm{~nm}$ as downstream and upstream wavelengths, respectively. The selected wavelengths give about a $1 \mathrm{~dB}$ bi-directional operation coupling loss. We added $2 \mathrm{~dB}$ extra loss at each transmitting side of the bi-directional transmissions to emulate the highest bi-directional operation coupling loss $(3 \mathrm{~dB})$. A Mach-Zehnder modulator driven by an arbitrary waveform generator generating 12.5 Gbaud PAM-4 signals with $2^{15}-1$ pseudorandom sequence symbols modulates either the downstream laser light at $1560 \mathrm{~nm}$ or the upstream laser light at $1530 \mathrm{~nm}$. The received optical signals are detected by a PIN photodiode with $0.7 \mathrm{~A} / \mathrm{W}$ responsivity; then the converted electrical signals are pre-amplified by a preamplifier with $15 \mathrm{~dB}$ of gain. The signals are then captured by an $80 \mathrm{GS} / \mathrm{s}$ real-time oscilloscope and are processed offline. A decision feedback equalizer is used to recover the PAM-4 signals. To ensure that the OWC system is eye-safe $(7 \mathrm{dBm})$, together with the $2 \mathrm{~dB}$ extra loss, the output power from port 2 of the circulators is set to $5 \mathrm{dBm}$ during the experiments. The weighting factors of the composite phase mask are optimized so that the downstream and upstream transmissions of a bi-directional link have an equal loss, and the optical power is equally broadcast to the user terminals. Without performing optical broadcasting, the measured bi-directional point-topoint optical link loss between the single-mode fibers at the SLM-based base station and one nomadic user terminal over $4 \mathrm{~m}$ indoor free space path is $19.8 \mathrm{~dB}$ (including the $3 \mathrm{~dB}$ bi-directional loss). The beam just before the steering mirror at the user terminal is observed to be slightly aberrated with a diameter of $12 \mathrm{~mm}$ after $4 \mathrm{~m}$, diverged from the collimated beam of diameter $7 \mathrm{~mm}$. The optical broadcasting to two user terminals introduces an additional $3 \mathrm{~dB}$ splitting loss. Using commercial transceiver modules with enough power budget, the pre-EDFA at the receiving end can be removed to reduce the cost of a user terminal.

Figure 4(b) shows the bit-error-rate (BER) measurements versus the received optical power (ROP) at $25 \mathrm{Gbit} / \mathrm{s}$ using PAM-4 modulation, together with typical eye diagrams. Due to the weighting factor optimization to equalize relative power of all the optical transmissions, the downstream and upstream transmissions of each broadcast link have equal losses $(22.8 \mathrm{~dB})$, and the broadcast power splits are equal. Therefore, it can be seen that the BER performances of the two broadcast downstream transmissions are almost identical, and each has a
1.9 $\mathrm{dB}$ power penalty compared with the back-to-back at a BER of $1 \times 10^{-4}$. The BER performance of the downstream transmission is also almost equivalent to the performance of its upstream transmission. Optical broadcasting to two user terminals has a $1.1 \mathrm{~dB}$ power penalty over the point-to-point operation (without the splitting loss) at a BER of $1 \times 10^{-4}$.

In summary, we have studied and successfully demonstrated wide FOV optical broadcasting for bi-directional indoor OWC at $25 \mathrm{Gbit} / \mathrm{s}$ using PAM- 4 over a range of $4 \mathrm{~m}$ using an SLMbased base station and nomadic user terminals using mirrorbased steering. Composite phase masks are constructed in a very simple way to perform optical broadcasting, as well as to support bi-directional transmissions at different designated wavelengths with a low additional coupling loss. The proposed system is designed to integrate with NG-PON2 fiber networks and broadcast TDM data to nomadic user terminals. With transceiver systems that have more power budget and optical loss improvement by optical aberration correction, we expect to achieve optical wireless broadcasting to a much larger number of nomadic user terminals.

Funding. Engineering and Physical Sciences Research Council (EP/P003990/1).

\section{REFERENCES}

1. T. Koonen, J. Lightwave Technol. 36, 1459 (2017).

2. D. C. O'Brien, "Optical wireless communications: current status and future prospects," in Proc. IEEE Summ. Top., Newport Beach (2016).

3. M. Maier, M. Levesque, and L. Ivanescu, IEEE Netw. 26, 15 (2012).

4. L.-Y. Wei, C.-W. Chow, G.-H. Chen, Y. Liu, C.-H. Yeh, and C.-W. Hsu, Opt. Express 27, 25072 (2019).

5. T.-C. Wu, Y.-C. Chi, H.-Y. Wang, C.-T. Tsai, Y.-F. Huang, and G.-R. Lin, Sci. Rep. 7, 11 (2017).

6. A. Gomez, K. Shi, C. Quintana, G. Faulkner, B. C. Thomsen, and D. O'Brien, J. Lightwave Technol. 34, 2510 (2016).

7. F. Feng, P. Sangwongngam, H. Chun, G. Faulkner, and D. O'Brien, in Conference on Lasers and Electro-Optics (Optical Society of America, 2019), paper SM2G.5.

8. D. Nesset, J. Lightwave Technol. 33, 1136 (2015).

9. F. Feng, I. H. White, and T. D. Wilkinson, J. Lightwave Technol. 31, 2001 (2013).

10. F. Feng, X. Jin, D. O’Brien, F. Payne, Y. Jung, Q. Kang, P. Barua, J. K. Sahu, S.-U. Alam, D. J. Richardson, and T. D. Wilkinson, Opt. Express 25, 13773 (2017).

11. N. Matsumoto, T. Inoue, T. Ando, Y. Takiguchi, Y. Ohtake, and H. Toyoda, Opt. Lett. 37, 3135 (2012).

12. F. Feng, I. H. White, and T. D. Wilkinson, J. Lightwave Technol. 32, 1239 (2014) 